Volume 2, Issue 2, April-June 2017, Pages: 181-182, DOI: http://dx.doi.org/10.19082/ah181

\title{
DESIGN AND DEVELOPMENT OF THE COMPREHENSIVE TRAFFIC INJURY REGISTRY AS THE NATIONAL PILOT IN NORTH-WEST PROVINCES OF IRAN
}

Homayoun Sadeghi-Bazargani ${ }^{1}$, Farmarz Pourasghar ${ }^{1}$, Bahram Samadirad ${ }^{2}$, Ali Tagizadiyeh ${ }^{1}$, Rouzbeh Rajaei ${ }^{1}$, Mousa Amiri ${ }^{3}$, Mashyaneh Haddadi ${ }^{4}$, Shahriyar Basirat ${ }^{3}$, Soudabeh Marin ${ }^{1}$, Farzad Rahmani ${ }^{1}$, Alireza Ala ${ }^{1}$,

Alireza Sadeghpour ${ }^{1}$, Ali Jannati ${ }^{5}$, Ali Meshkini ${ }^{1}$, Ghaffar Shokouhi ${ }^{1}$, Mohammad Navali ${ }^{1}$, Hamid Soori ${ }^{6}$, Alireza Moghisi ${ }^{4}$, Shahram Habibzadeh 7 , Mohammad Delirrad ${ }^{8}$, Djafarsadegh Tabrizi ${ }^{4}$, Ahmad Kousha ${ }^{4}$, Ali Ebadi ${ }^{1}$, Reza Deljavan ${ }^{1}$, Seyed Hossein Ojaghi ${ }^{1}$, Saeid Saghatizadeh ${ }^{1}$, Mahnaz Asadi Zadeh ${ }^{1}$

1: Tabriz University of Medical Sciences, Tabriz, Iran

2: Forensic Medicine Research Center, Tehran, Iran

3: Traffic Police of Iran, NAJA, Tehran, Iran

4: Ministry of Health \& Medical Education, Tehran, Iran

5: Maragheh University of Medical Sciences, Maragheh, Iran

6: Shahid Beheshti University of Medical Sciences, Tehran, Iran

7: Ardabil University of Medical Sciences, Ardabil, Iran

8: Urmia University of Medical Sciences, Urmia, Iran

\section{Correspondence:}

Homayoun Sadeghi-Bazargani, Tel: +98.9144027218, E-mail: homayoun.sadeghi@gmail.com

\section{TYPE OF ARTICLE: CONFERENCE ABSTRACT}

\begin{abstract}
Introduction: Many people think that putting data about traffic injuries into a computer or extracting traffic related data from a hospital information system could be called a traffic injury registry. No officially approved and comprehensive traffic injury registry existed in Iran before the Ministry of Health in Iran decided to designate an Iranian traffic knowledge development trustee as the responsible organization to design and develop the comprehensive traffic injury registry.

Methods: The Ministry of Health Secretary for Research jointly with Ministry of Health Secretary for Health and Treatment jointly appointed an Iranian traffic knowledge development trustee headed by Tabriz Traffic Injury Research Center to design and develop the comprehensive traffic injury registry (CTIR) as a national pilot to be implemented initially, in four provinces in northwest Iran and to be run for 4 years, before finalizing it for potential extension to other parts of the country. The health system, forensic medicine organization and traffic police of Iran are the three major sectors involved in this research. The registry is organized in 4 committees (Steering committee; Technical \& Engineering committee; Methodology committee; Administrative committee) and 14 operational teams.

Results: The CITIR was designed for traffic injury related data collections at five serial/parallel stations as 1: Traffic crash scene station 2- EMS transfer station 3- Emergency department station 4- Hospital ward station 5- Forensic medicine organization station. The complementary information is added from patient records to the registry server also. All the information is encrypted and linked through appropriate algorithms. The data collection staff at stations 3 and 5 are physicians and others are either emergency care technicians, nurses or non-medical staff. The data collection tools were prepared by an expert panel and followed validity and reliability assessment methodologies. Other than classical analytical methods, supervised pattern analysis methods and spatial analytical processes are applied for reporting structure.

Conclusion: Partial implementation of the designed CITIR, reveals potential applicability of this registry in Iran, however, it needs to be evaluated continuously and improved in a mid-term investigation before its extended implementation.
\end{abstract}

\section{Abstracts of First National Congress of Medical Informatics, Mashhad, Iran, February 2017}

(C) 2017 The Authors. This is an open access article under the terms of the Creative Commons Attribution-NonCommercialNoDerivs License, which permits use and distribution in any medium, provided the original work is properly cited, the use is non-commercial and no modifications or adaptations are made. 
KEYWORDS: Traffic injuries, Road safety, Trauma, Traffic injury registry, Iran 